\title{
Complementary Atom Probe Tomography and Electron Microscopy of Oxidation of Ni-base Alloys in High-Temperature Water Environments
}

\author{
Daniel K. Schreiber, Matthew J. Olszta and Stephen M. Bruemmer
}

Energy and Environment Directorate, Pacific Northwest National Laboratory, Richland, WA, USA

Materials degradation in aggressive environments is a complex, scientifically rich area of research that is also of vital importance to many industrial applications. One such example is stress-corrosion cracking (SCC) of Ni-base alloys in the high-temperature, hydrogenated water environments found within nuclear reactors. [1] In this work, we show how advanced microscopy techniques can enhance the fundamental understanding of material degradation processes in these challenging environments and especially how the complementary aspects of transmission electron microscopy (TEM) and atom probe tomography (APT) can provide novel insights into these phenomena.

Materials for these studies consisted of polished coupons of commercial and model Ni-base alloys exposed to simulated pressurized water reactor primary water environments $\left(320-360^{\circ} \mathrm{C}\right.$ hydrogenated water) for 500-3500 h. [2] Alloys with low Cr concentrations (e.g. Ni-17Cr-9Fe alloy 600) exhibited intergranular oxidation (Fig 1a). In this first example, separate samples were used for TEM and APT characterization. Electron diffraction (not shown) revealed the primary oxide phase to be unoriented MO (rock-salt type) oxide. STEM-EDS mapping (Fig 1b) established that the oxides were Cr-rich and depleted of $\mathrm{Fe}$ and $\mathrm{Cr}$. The 3D microstructure of the oxide was determined near its termination by APT using isoconcentration surfaces (Fig 1c). The oxides, forming fingerlike protrusions down the grain boundary, were interconnected up to the terminal point. Compositional analyses (Figs 1d-e) confirmed the Cr-rich nature of the oxides and also revealed very strong $\mathrm{Cr}$ depletion extending for over $10 \mathrm{~nm}$ perpendicular to the grain boundary, which was not apparent by TEM, possibly due to image projection.

Additional analyses have been performed using directly correlated TEM/APT examinations. This approach is beneficial as it permits direct correlation of the oxide crystal structure (TEM) with the 3D oxide morphology and composition (APT) while also improving the accuracy of the APT reconstruction scaling. A representative analysis is presented in Fig. 2 of intragranular oxidation in alloy 690 (Ni-30Cr9Fe). [3] Electron diffraction identified 3 distinct oxide phases: $\mathrm{M}_{3} \mathrm{O}_{4}$ at the apex, $\mathrm{Cr}_{2} \mathrm{O}_{3}$, and a deep linear penetration of oriented $\mathrm{MO}$, possibly following a matrix dislocation. In contrast to the interconnected finger-like oxides in alloy 600, the MO penetration in alloy 690 consisted of discrete clusters of Cr-rich oxide (Fig 2c,e) that did not extend throughout the cylindrical volume of material. This difference in oxide morphology suggests that the oxidation mechanism may be different for the two materials systems and requires additional investigations.

References:

[1] L.E. Thomas and S.M. Bruemmer, Corrosion 56(6) (2000), p572-587.

[2] D.K. Schreiber et al, Microscopy \& Microanalysis (2013), in press.

[3] M.J. Olszta et al, Advanced Materials \& Processes 170(4) (2012), p17-21.

[4] The authors acknowledge funding from the Department of Energy, Office of Basic Energy Sciences under contract DE-AC06-76RLO 1830. The APT research was accomplished at the EMSL, a national scientific user facility sponsored by the Department of Energy's Office of Biological and Environmental Research and located at Pacific Northwest National Laboratory. 

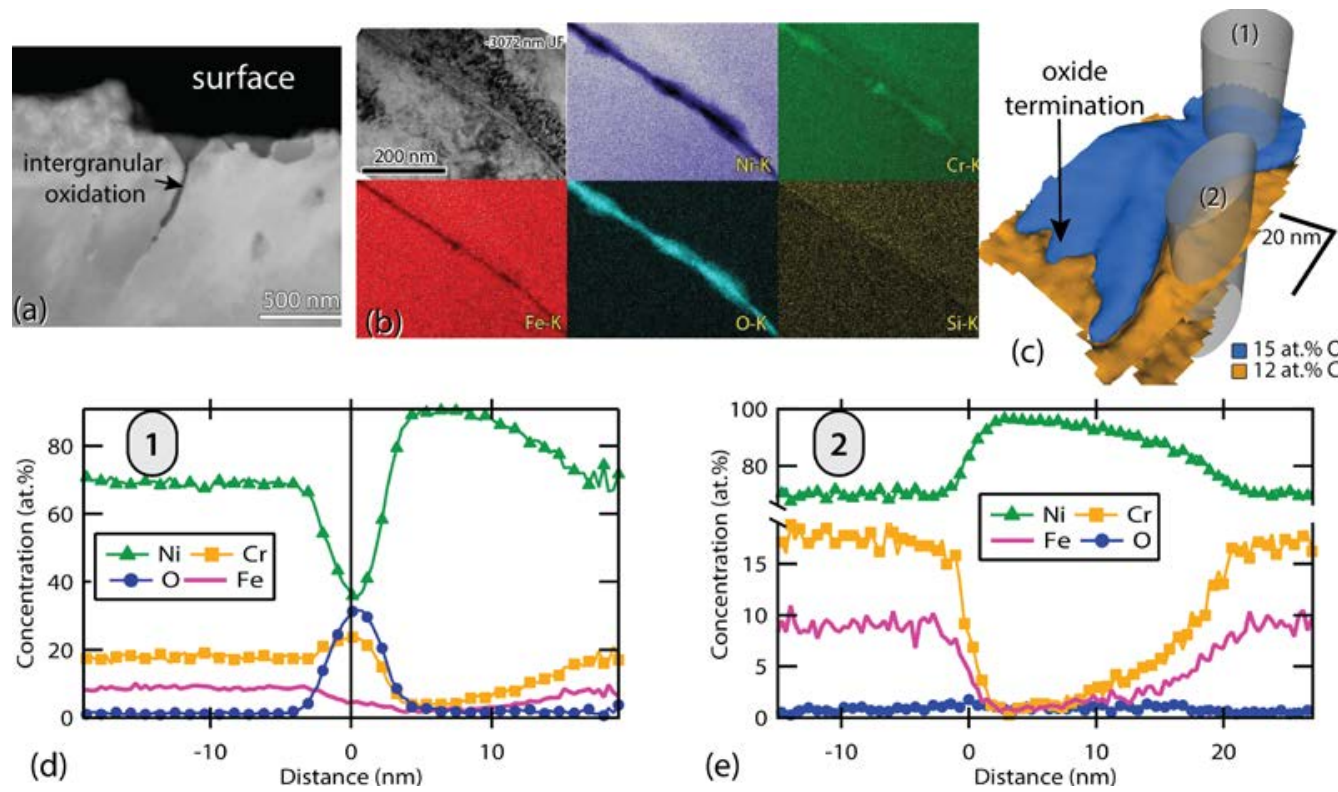

Figure 1. (a) SEM of intergranular oxidation in alloy 600. (b) STEM EDS mapping reveals a continuous $\mathrm{Cr}$-rich oxide depleted of $\mathrm{Ni}$ and $\mathrm{Fe}$. (c) Isoconcentration surfaces from an APT reconstruction at the oxide termination reveal a 3D interconnected finger-like oxide microstructure while $1 \mathrm{D}$ concentration profiles (d)-(e) confirm the Cr-rich oxide and strong depletion of $\mathrm{Cr}$ and Fe nearby.
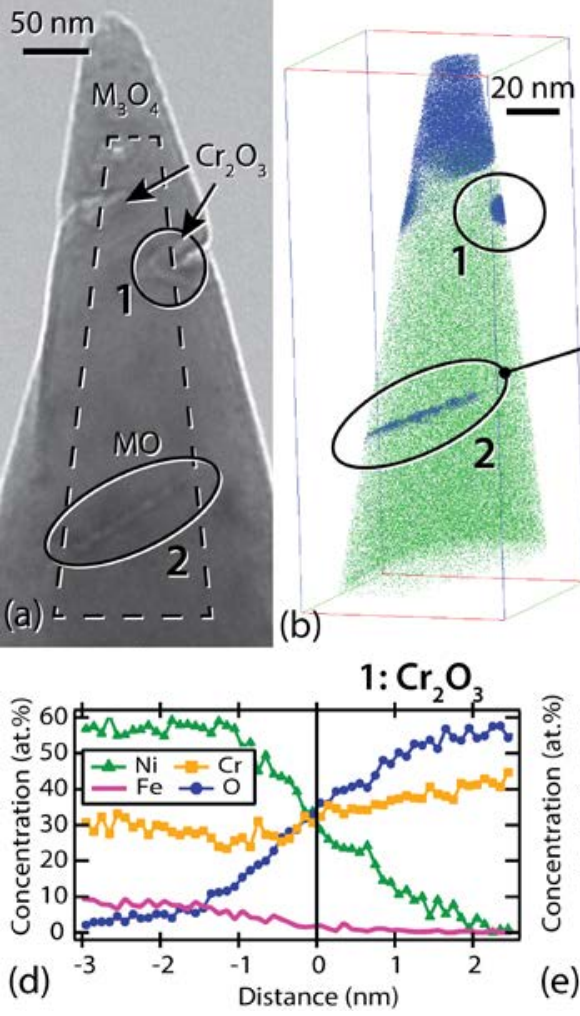
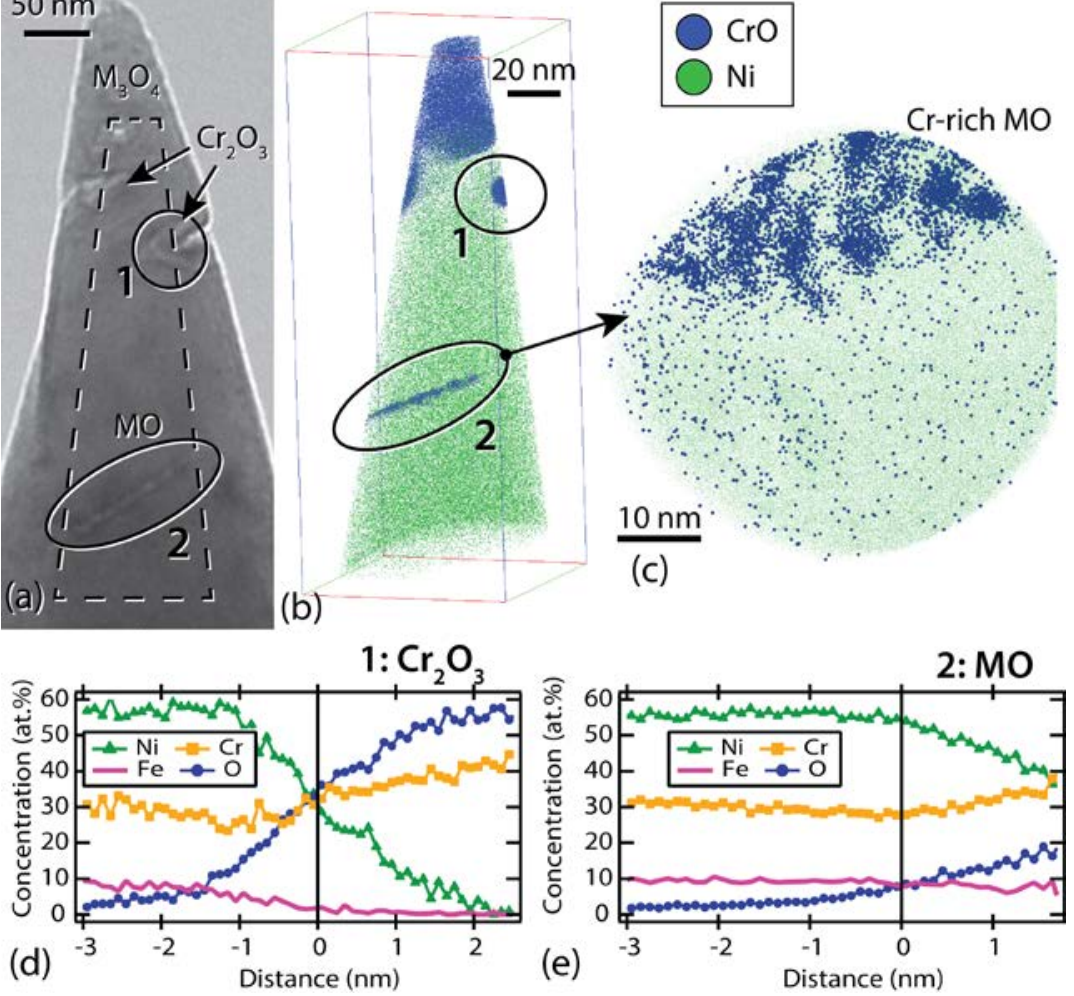

\section{- $\mathrm{Ni}$}

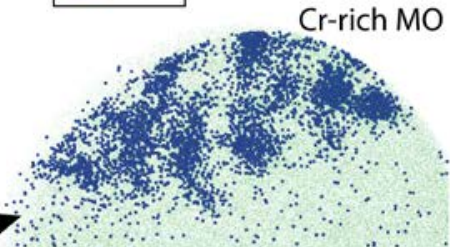

$\because$
$\because$
$\because$

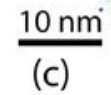

(c)

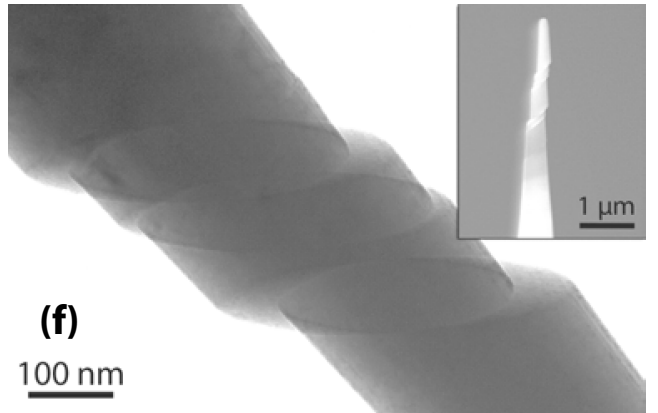

Figure 2. Directly correlated TEM and APT of intragranular oxidation in alloy 690. The reconstructed APT volume (b) is outlined by a dashed line in the BF TEM image (a). $T$ hree distinct oxide phases were observed: $\mathrm{M}_{3} \mathrm{O}_{4}, \mathrm{Cr}_{2} \mathrm{O}_{3}$ and $\mathrm{MO}$ phase. A PT revealed the MO-phase (2) consisted of discrete clusters of $\mathrm{Cr}$-rich oxide and proximity histograms (d)-(e) showed all of the oxides were Cr-rich, with nearly stoichiometric $\mathrm{Cr}_{2} \mathrm{O}_{3}$ measurements (1). (f) BF TEM and (inset) SEM images of a Ni-5Al binary alloy APT specimen that sheared along slip planes during APT testing without fracturing. This suggests catastrophic fracture can be preceded by severe specimen deformation that typically goes undetected. 